(D) Check for updates

Cite this: Polym. Chem., 2022, 13. 479

Received 9th November 2021, Accepted 17th December 2021

DOI: 10.1039/d1py01510a

rsc.li/polymers

\section{Putting the RAFT in GRAFT: intermolecular graft exchange between bottlebrush polymers using reversible addition-fragmentation chain transfer $\dagger$}

\author{
Satu Häkkinen, (D) a Billy Dyer, ${ }^{a}$ Andrew Kerr ${ }^{a}$ and Sébastien Perrier (D)*a,b
}

\begin{abstract}
A versatile synthetic methodology is presented for the preparation of graft copolymers with mixed graft distributions using reversible addition-fragmentation chain transfer (RAFT). The approach harnesses the ability of $Z$ group-tethered grafts to fragment off the backbone to facilitate intermolecular graft exchange reactions between distinct starting materials.
\end{abstract}

The discovery and industrious development of controlled polymerisation techniques have allowed the construction of macromolecules with intricate architectures and functionalities. ${ }^{1}$ Today, architectural design is an elemental step in adjusting polymer properties to suit its application. Further exploration of new synthetic routes is needed to expand the horizons of functional polymers. ${ }^{2,3}$

Branched architectures have been widely studied for their intriguing solution and bulk properties arising from their high density, reduced interchain penetration and entanglements, and conformational constraints imposed by their branching points. ${ }^{4,5}$ Amongst them, graft copolymers continue to be of interest to material scientists. ${ }^{6-8}$ The field has stemmed three synthetic strategies for their preparation, all of which may be used in conjunction with one or more polymerisation techniques. These include polymerisation of macromonomers (grafting through), conjugation of grafts to a substrate (grafting to), and polymerisation of grafts from initiating sites on a substrate (grafting from). ${ }^{7}$

The grafting from approach is a versatile synthetic strategy from a chemical and a mechanistic standpoint when conducted as a RAFT polymerisation. ${ }^{9-12}$ In RAFT grafting from polymerisations initiating sites (i.e., RAFT agents) may be tethered to a substrate via the reinitiating $\mathrm{R}$ group or the stabilising $\mathrm{Z}$ group, giving rise to two mechanistically different reac-

\footnotetext{
${ }^{a}$ Department of Chemistry, University of Warwick, Coventry CV4 7AL, UK. E-mail:s.perrier@warwick.ac.uk

${ }^{b}$ Warwick Medical School, University of Warwick, Coventry CV4 7AL, UK $\dagger$ Electronic supplementary information (ESI) available: Experimental details and characterisation data. See DOI: 10.1039/d1py01510a
}

tions with each having their benefits and limitations. ${ }^{9}$ In the $\mathrm{R}$ group approach propagating graft radicals remain covalently bound to the substrate, resulting in similar reaction mechanics to grafting from polymerisations conducted with other controlled polymerisation techniques. The $\mathrm{Z}$ group reaction mechanism is unique to RAFT polymerisation and akin to reversible grafting to radical reactions. In the $\mathrm{Z}$ group approach - also known as the transfer to approach ${ }^{13}$ - grafts fragment off their graft sites to propagate and may diffuse freely in the reaction medium. We hypothesised that the graft fragmentation should lead to intermolecular graft exchange if graft radicals were able to diffuse away from their original graft sites and close to those on another molecule (Scheme 1). Building on this feature, the $\mathrm{Z}$ group approach could be used to exchange distinct grafts in a mixture of graft copolymers to yield hybrid products, giving access to heterograft structures in a simple manner. While densely grafted heterograft copolymers may also be conveniently produced via the grafting through strategy, these polymerisations can suffer from poor control when conducted using RAFT and targeting long backbones due to steric hindrance congestion near the propagating and dormant chain-ends. ${ }^{14}$ It is therefore useful to find alternative ways to achieve these structures.

We present two convenient routes through which graft copolymers with mixed graft distributions - such as heterograft copolymers - may be prepared using the $\mathrm{Z}$ group mechanism. The first route involves mixing two or more structurally different graft copolymers in solution and subsequent initiation to induce graft interchange. The exchange may also be conducted using a mixture of graft copolymers and linear polymers capable of forming a chain radical. In the second approach a linear polymer with chain transfer agent-functionalised side groups (pCTA) and a linear polymer with a RAFT end-group are reacted to graft the linear chains to the PCTA using radical reactions.

A library of graft copolymers was prepared for the graft exchange study using a three-step synthetic protocol. In short, RAFT polymerisation and post-modification of poly(2-hydroxyethyl acrylate) (pHEA) was conducted to give a functionalised 

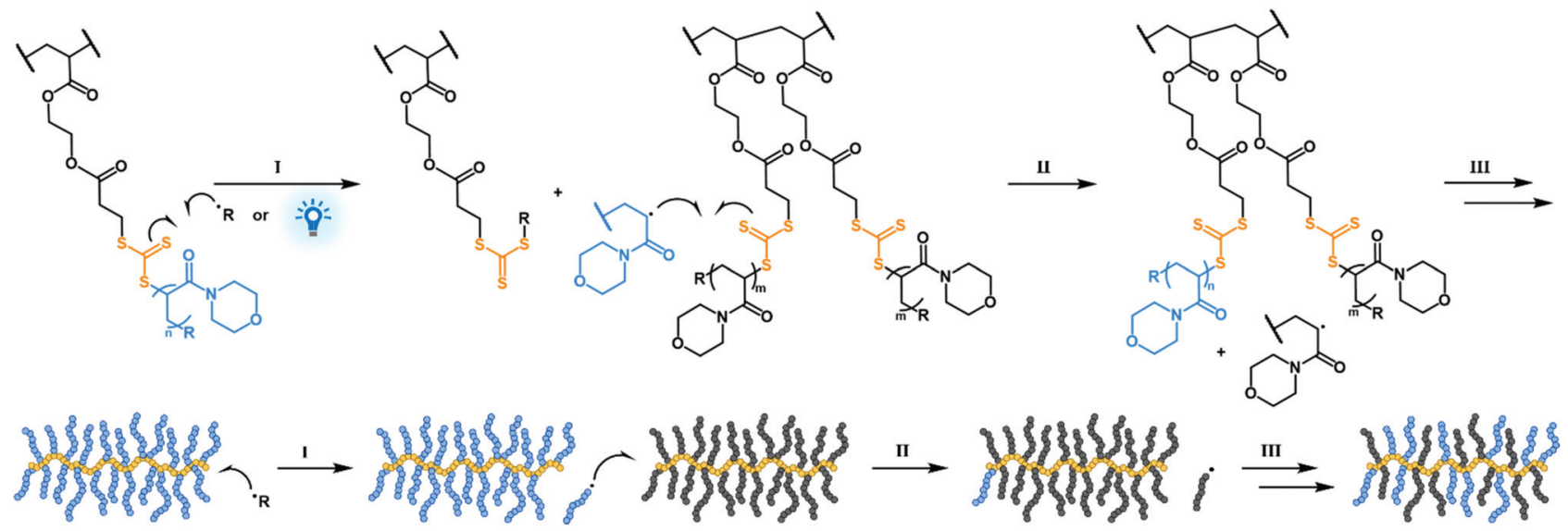

Scheme 1 Proposed graft exchange mechanism. Radical addition or photolysis of a grafting site leads to graft fragmentation (I) and intermolecular addition to another RAFT agent, consequently fragmenting the original graft (II). Repeated events (III) give a homogeneous product.

pHEA with 3-((((1-methoxy-1-oxopropan-2-yl)thio)carbonothioyl)thio)propanoic acid (MPPATC) tethered to the hydroxy groups via the $\mathrm{Z}$ group. Using such pCTAs as photoiniferters, ${ }^{15}$ a series of graft copolymers was prepared by polymerising 4-acryloylmorpholine (NAM) via the $\mathrm{Z}$ group approach under blue light irradiation. The products were isolated through repeated precipitations to remove any residual monomer and terminated linear polymers and characterised with size-exclusion chromatography (SEC) and ${ }^{1} \mathrm{H}$ NMR spectroscopy. A selection of linear polymers was prepared via photoiniferter RAFT polymerisations of NAM to study the linear chain grafting approach. (ESI†: full synthesis and characterisation details of all materials.)

To test our hypothesis of intermolecular graft exchange taking place upon fragmentation of $\mathrm{Z}$ group tethered grafts, a reaction was conducted between pHEA $_{23}$-graft-pNAM ${ }_{10}$ and pHEA $_{23}$-graft-NAM ${ }_{87}$ under typical RAFT polymerisation conditions using an equal mass of each polymer and dimethyl 2,2'azobis(2-methylpropionate) (V-601) initiator $\left([\mathrm{CTA}] /[\mathrm{I}]_{0}=20\right)$ at $75^{\circ} \mathrm{C}$ in dioxane. The polymers comprised identical backbones (DP 23) but different graft lengths (DPs 10 and 87) and were therefore expected to exhibit a unimodal molecular weight distribution (MWD) upon a successful exchange. Samples were withdrawn throughout the reaction for SEC analysis. The chromatogram of a sample taken before initiation showed two distinct MWDs, corresponding to pHEA $_{23}$-graft-pNAM 10 with short grafts at the lower end and pHEA $_{23}$-graft-pNAM ${ }_{87}$ with longer grafts at the higher end of the molecular weight range (Fig. 1A). Changes in the MWDs after 15 minutes indicated a successful initiation of intermolecular graft exchange. This change became more apparent with increasing reaction time as the larger and smaller species continued to shift towards lower and higher molecular weights, respectively. After $3 \mathrm{~h}$ the exchange was sufficient to result in a nearly uniform MWD with $M_{\mathrm{n}, \mathrm{SEC}}=$ $39600, D=1.46$, and a subtle peak split still visible. Roughly $4 \mathrm{wt} \%$ of terminated grafts were formed over $15 \mathrm{~h}$, corresponding to the expected $5 \mathrm{~mol} \%$ termination due to the added initiator ${ }^{16}$ and resulting in a reduced grafting density. The main distribution exhibited subtle asymmetry after the reaction due to different degrees of graft termination in the preparation of the starting materials (Table S3†), which lead to the two polymers having slightly different grafting densities and therefore different chain volumes despite having indistinguishable graft length distributions after the exchange. Overall, the data indicated a successful graft exchange.

The reaction was repeated in the absence of exogenous initiator by employing the side group trithiocarbonate as a photoiniferter. $^{15} \mathrm{~A}$ blue light induced reaction between pHEA $_{23}$-graft-pNAM ${ }_{10}$ and pHEA $_{23}$-graft-pNAM ${ }_{87}$ at $40-50{ }^{\circ} \mathrm{C}$ showed a nearly identical transformation of the MWD over $15 \mathrm{~h}$, however the apparent rate of graft exchange was slower than in the initiator-driven reaction (Fig. S12†). The slower reaction rate was ascribed to a reduced frequency of successful graft detachment events due to a slower rate of radical formation, cage reactions, and/or slower radical diffusion. Repeated attempts at $20-30{ }^{\circ} \mathrm{C}$ resulted in no apparent exchange over $15 \mathrm{~h}$, likely due to a reduced bond dissociation and/or diffusivity.

One of the advantages of the presented synthetic strategy is that the graft distribution may be adjusted with reaction stoichiometry. This modularity could be particularly advantageous in studies involving large polymer libraries in which one or multiple properties, such as polymer aspect ratio, rigidity, charge density, or a functionality are systematically varied. To this end, photoiniferter graft exchange reactions were conducted between pHEA $_{133}$-graft-pNAM ${ }_{8}$ and pHEA $_{133}$-graftpNAM $_{29}$ in $2: 1,1: 1$, and $1: 2$ mass ratios of the two polymers to yield three products with distinct hydrodynamic volumes (Fig. 1B). Some termination was observed ( $\leq 7 \mathrm{wt} \%)$, the amount of which increased with increasing average graft length. A reaction between graft copolymers with both different backbone and graft lengths, pHEA $_{23}$-graft-pNAM 10 and pHEA $_{300}$-graft-pNAM ${ }_{51}$, resulted in a mixture of polymers with similar graft size distributions but dissimilar hydrodyn- 
A

A

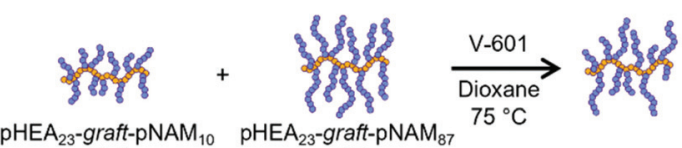

pHEA $_{23}-$ graft

(1b)

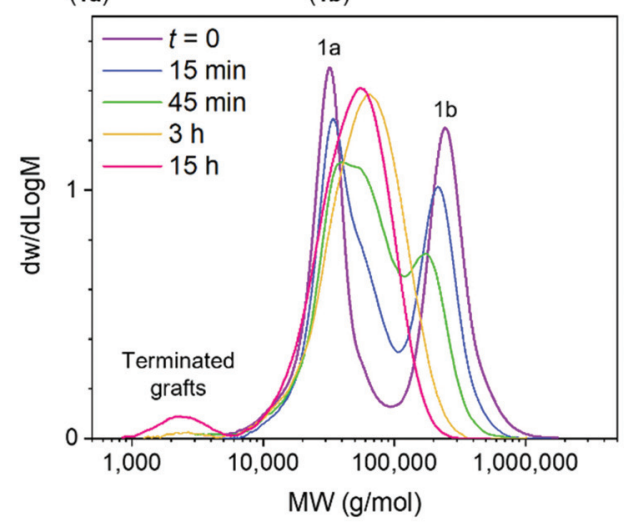

B

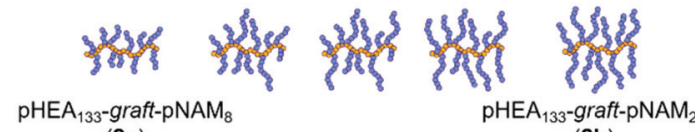

(2a)

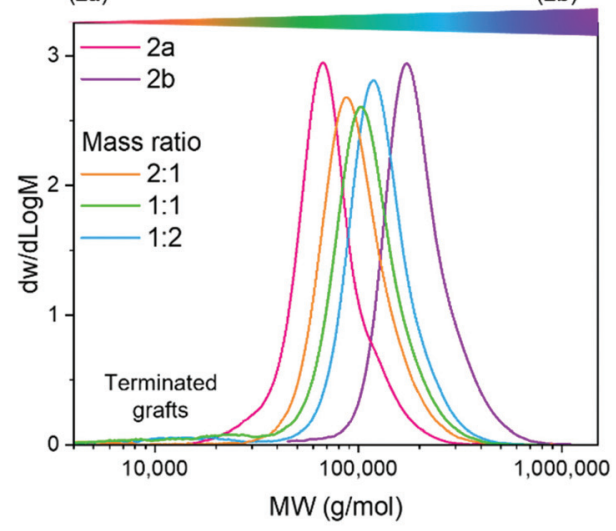

Fig. 1 (A) SEC analysis of a graft exchange reaction between $\mathrm{pHEA}_{23}$ graft-pNAM 10 and $\mathrm{pHEA}_{23}$-graft-pNAM ${ }_{87}$. (B) SEC analysis of $\mathrm{pHEA}_{133^{-}}$ graft-pNAM $8, \mathrm{pHEA}_{133}$-graft-pNAM 29 , and the products of exchange reactions carried out using $2: 1,1: 1$ and 1:2 mass ratios of the two polymers. Analysis was performed in DMF with DRI detection and PMMA calibration.

amic volumes (Fig. S10 and S11 $\dagger$ ). While these reactions were performed in the absence of monomer, the exchange could alternatively be carried out as a block extension of the original grafts.

Grafts may also be exchanged for linear chains capable of forming a chain radical, providing a versatile functionalisation strategy. This was demonstrated by exchanging grafts of $\mathrm{pHEA}_{23}$-graft-pNAM ${ }_{10}$ for pyrene-functional linear $\mathrm{pNAM}_{15}$ under blue light irradiation. Equimolar amounts of linear chains and grafts were used in an attempt to exchange $50 \%$ of the original grafts for pyrene-functional grafts. The reaction was monitored by SEC using UV detection at a $265 \mathrm{~nm}$ wavelength, at which pyrene has a strong absorption but trithiocarbonate groups absorb only weakly. The data showed a gradual increase in the UV absorption of the graft copolymer relative to the linear polymer, reaching $45 \%$ of total absorption over $24 \mathrm{~h}$ and confirming a successful functionalisation of the bottlebrush polymer with a fluorescent probe (Fig. 2).

The $\mathrm{Z}$ group approach reaction mechanism was also used to access the graft copolymer architecture by reacting linear pNAM chains with a pCTA. Using this strategy, graft copolymers may be prepared from a mixture of linear polymers in a similar fashion to previously reported grafting to reactions with polymeric radicals. ${ }^{17}$ Due to the ability of grafts to continuously fragment off the backbone, the reaction was expected to reach an equilibrium wherein the addition and fragmentation of grafts takes place at equal rates. Therefore, the reactions would result in a mixture of graft copolymers and linear chains which would need to be separated to isolate the desired

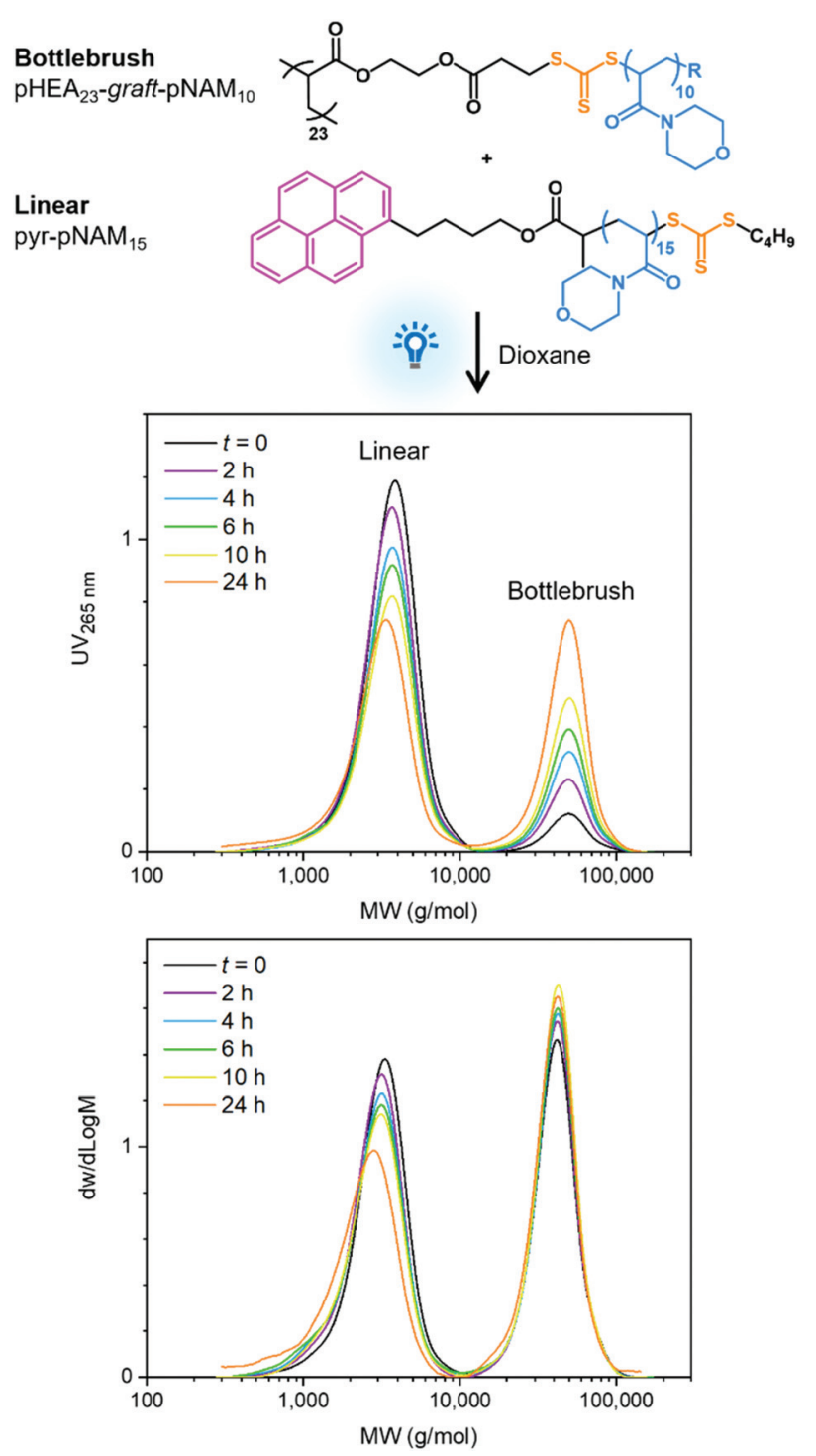

Fig. 2 SEC data shows exchange of $\mathrm{pHEA}_{23}$-graft-pNAM 10 grafts for pyrene-functional linear $\mathrm{pNAM}_{15}$ chains. Analysis was conducted in $\mathrm{CHCl}_{3}$ with DRI and $\mathrm{UV}_{265 \mathrm{~nm}}$ detection and PMMA calibration. 
product. The ratio of linear chains to backbone CTAs in the reaction was expected to determine the number of linear chains attached to the backbone.

To this end, $\mathrm{pNAM}_{44}$ was reacted with $\mathrm{pCTA}_{300}$ in a $1: 1$ molar ratio of linear chains to backbone CTAs in the presence of V-601 $\left([\mathrm{CTA}]_{\mathrm{o}} /[\mathrm{I}]_{0}=20\right)$ in dioxane at $75{ }^{\circ} \mathrm{C}$. SEC indicated a rapid increase in the hydrodynamic volume of $\mathrm{PCTA}_{300}$ within the first $30 \mathrm{~min}$ as linear chains were grafted to the polymer (Fig. 3A). The reaction was fast, and samples taken at longer reaction times indicated very little change in the MWD as the reaction reached an equilibrium. The fraction of grafted chains was estimated by monitoring the formation of UVactive, end-group-derived by-products resulting from chain grafting to the backbone. The initial molar ratio of $\mathrm{pNAM}_{44}$ to

A
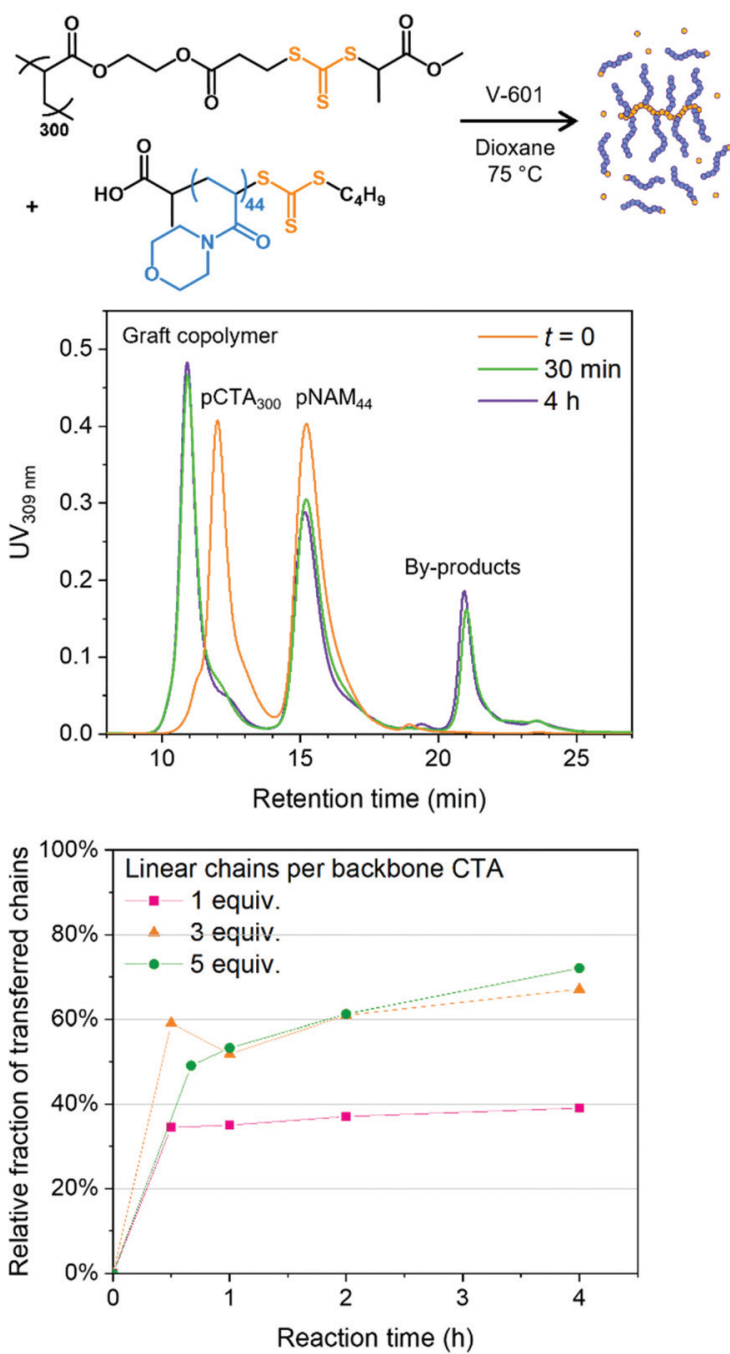

Fig. 3 (A) SEC data for the transfer of $\mathrm{pNAM}_{44}$ to $\mathrm{pCTA}_{300}$ in the presence of $\mathrm{V}-601$ at $75{ }^{\circ} \mathrm{C}$ in dioxane. Auv was normalised for each dataset. Analysis was conducted in DMF with DRI and $U_{309 \mathrm{~nm}}$ detection and PMMA calibration. (B) Relative fraction of transferred linear chains ( $\left.f_{\mathrm{Re}, \mathrm{UV}}\right)$, descriptive of the grafting density, plotted against reaction time in reactions with $1: 1,3: 1$, and $5: 1$ pNAM to backbone CTA ratios. backbone CTAs was estimated from their respective peak areas $A_{\mathrm{UV}, \mathrm{pNAM}}$ and $A_{\mathrm{UV}, \mathrm{pCTA}}$ as

$$
R_{\mathrm{pNAM} / \mathrm{CTA}}=\frac{A_{\mathrm{UV}, \mathrm{pNAM}}}{A_{\mathrm{UV}, \mathrm{pCTA}}},
$$

However, the molar absorptivities of backbone CTAs and pNAM $_{44}$ were not known to be equal and the relative areas were only used as an approximation. ${ }^{18}$ The relative fraction of grafted linear chains, which is descriptive of the grafting density, was calculated using the peak area of the by-products $\left(A_{\text {UV,CTA }}\right)$ as

$$
f_{\mathrm{Rel}, \mathrm{UV}}=R_{\mathrm{pNAM} / \mathrm{CTA}} \times \frac{A_{\mathrm{UV}, \mathrm{CTA}}}{A_{\mathrm{UV}, \mathrm{CTA}}+A_{\mathrm{UV}, \mathrm{pNAM}}}
$$

by assuming the molar absorptivities of the by-products to roughly equal that of $\mathrm{pNAM}_{44}$. The reaction reached a plateau after some $39 \%$ of the original $\mathrm{R}$ groups of MPPATC had been exchanged for a $\mathrm{pNAM}_{44}$ chain, falling short of the theoretical maximum of $50 \%$. The data was in a reasonably good agreement with the $31 \%$ grafting calculated from RI vs. RT data (Table $\mathrm{S} 7 \dagger$ ).

An excess of linear chains was used in subsequent reactions to target higher grafting densities. Steric shielding effects near the reactive sites were anticipated to set a practical upper limit for the number of grafts per backbone. Grafting was carried out using $3: 1$ and 5:1 molar ratios of pNAM $_{44}$ to backbone CTAs, expecting to reach $f_{\text {Rel,Uv }}=75 \%$ and $83 \%$, respectively, but observing $67 \%$ and $72 \%$ after $4 \mathrm{~h}$ (Fig. 3B). The data suggested that even with a large excess of linear polymer roughly $30 \%$ of backbone repeating units remained without a graft.

While this approach limits the achievable grafting density, it gives an excellent control over graft dispersity. Three grafting reactions between pCTA $_{300}$ and pNAM (DP 20, 30, and 44) were conducted to compare the MWDs of the grafted chains after cleaving them off the backbone. Grafting was performed under blue light irradiation over $1.5 \mathrm{~h}$ with an equimolar ratio of linear chains to backbone CTAs, resulting in a 30-35\% grafting density. After isolating the graft copolymers from linear chains through repeated precipitations, the grafts were fragmented off the backbone with blue light irradiation in the presence of 1-ethylpiperidine hypophosphite ${ }^{19}$ and analysed by SEC (Fig. 4). The cleaved grafts retained the molecular weight and dispersity of the original linear polymer well, and a clear distinction could be made between the three graft lengths after cleavage. In contrast, grafts polymerised through the $\mathrm{Z}$ group approach exhibited much higher dispersities $(\nexists=1.58-1.98)$ regardless of the backbone or graft length. The poor control was to be expected due to chain transfer to the graft sites being hindered by steric shielding effects and the fast propagation rate of NAM. ${ }^{16,20}$ The new approach seems beneficial when low dispersity grafts are preferred over high grafting densities or when the selected materials cannot be synthesised through grafting from polymerisations. The strategy is applicable to linear polymers prepared through other polymeris- 


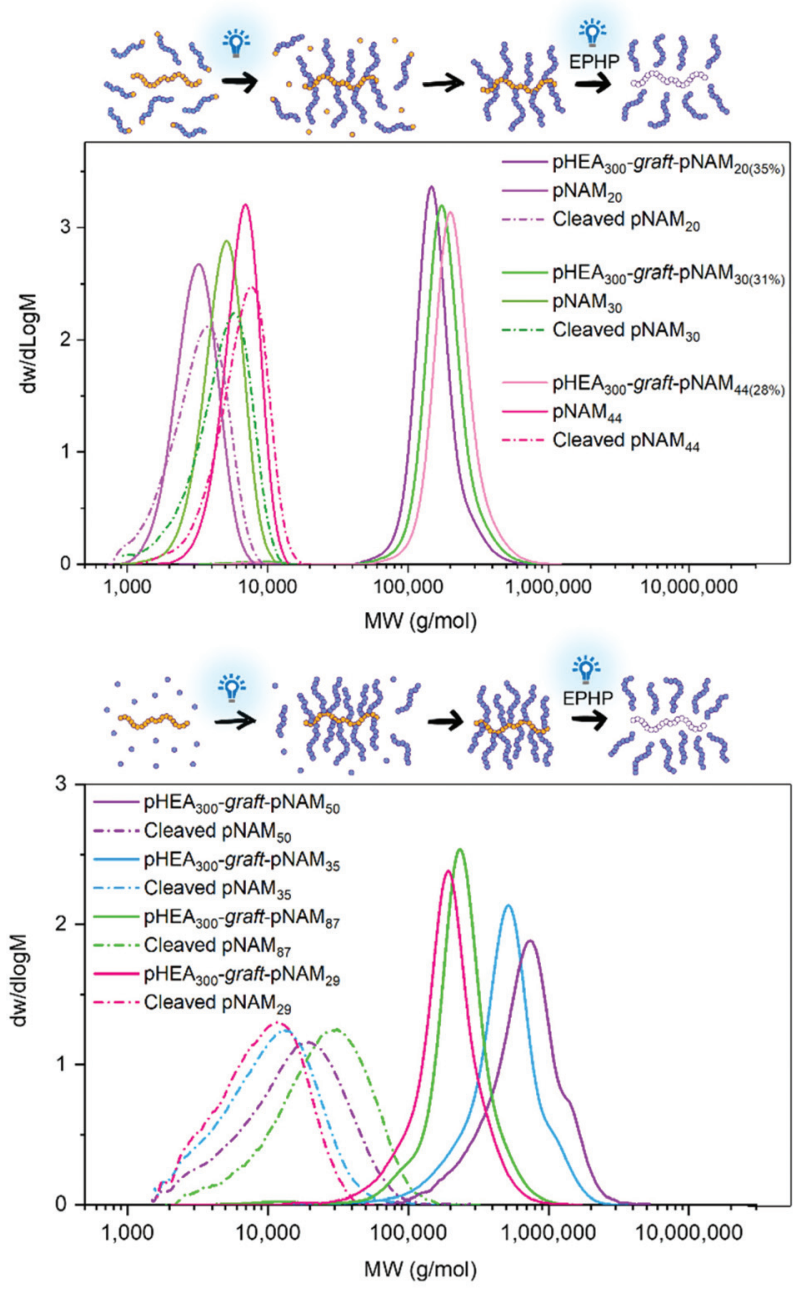

Fig. 4 SEC data of grafts cleaved off polymers synthesised via grafting of linear pNAM to pCTA 300 (top) and the $Z$ group approach (bottom). Analysis was conducted in DMF with DRI detection and PMMA calibration.

ation methods, provided that they carry a suitable radicalforming functionality.

In conclusion, the $\mathrm{Z}$ group approach was used to realise two new synthetic strategies that may be adapted as a convenient way to construct heterograft copolymers and other mixed graft distributions. In applying $\mathrm{Z}$ group grafting strategies, attention should be paid to the radical flux to minimise graft termination which may generally be expected to increase with increasing graft length due to steric shielding effects. Parameters such as reaction temperature, viscosity, concentration, ${ }^{21}$ and solvent quality ${ }^{22}$ may be used to optimise each system. In reactions employing various monomer families (e.g., methacrylic and acrylic monomers) the relative reactivities and radical stabilities of each should be taken into consideration. The versatile UV absorption characteristics and reactivities of RAFT agents may be explored further to develop more elegant grafting strategies. The scope of our work may be expanded beyond RAFT chemistry by employing polymers pre- pared through other polymerisation techniques carrying radical forming functionalities.

\section{Conflicts of interest}

There are no conflicts to declare.

\section{Acknowledgements}

Lubrizol is acknowledged for the provision of scholarships ( $\mathrm{SH}$ and $\mathrm{AK})$.

\section{Notes and references}

1 R. B. Grubbs and R. H. Grubbs, Macromolecules, 2017, 50, 6979.

2 A. S. Abd-El-Aziz, M. Antonietti, C. Barner-Kowollik, W. H. Binder, A. Böker, C. Boyer, M. R. Buchmeiser, S. Z. D. Cheng, F. D'Agosto, G. Floudas, H. Frey, G. Galli, J. Genzer, L. Hartmann, R. Hoogenboom, T. Ishizone, D. L. Kaplan, M. Leclerc, A. Lendlein, B. Liu, T. E. Long, S. Ludwigs, J. F. Lutz, K. Matyjaszewski, M. A. R. Meier, K. Müllen, M. Müllner, B. Rieger, T. P. Russell, D. A. Savin, A. D. Schlüter, U. S. Schubert, S. Seiffert, K. Severing, J. B. P. Soares, M. Staffilani, B. S. Sumerlin, Y. Sun, B. Z. Tang, C. Tang, P. Théato, N. Tirelli, O. K. C. Tsui, M. M. Unterlass, P. Vana, B. Voit, S. Vyazovkin, C. Weder, U. Wiesner, W. Y. Wong, C. Wu, Y. Yagci, J. Yuan and G. Zhang, Macromol. Chem. Phys., 2020, 221, 2000216.

3 K. Parkatzidis, H. S. Wang, N. P. Truong and A. Anastasaki, Chem, 2020, 6, 1575.

4 S. E. Seo and C. J. Hawker, Macromolecules, 2020, 53, 3257.

5 G. Polymeropoulos, G. Zapsas, K. Ntetsikas, P. Bilalis, Y. Gnanou and N. Hadjichristidis, Macromolecules, 2017, 50, 1253.

6 M. Müllner and A. H. E. Müller, Polymer, 2016, 98, 389.

7 G. Xie, M. R. Martinez, M. Olszewski, S. S. Sheiko and K. Matyjaszewski, Biomacromolecules, 2019, 20, 27.

8 Z. Li, M. Tang, S. Liang, M. Zhang, G. M. Biesold, Y. He, S.-M. Hao, W. Choi, Y. Liu, J. Peng and Z. Lin, Prog. Polym. Sci., 2021, 116, 101387.

9 J. C. Foster, S. C. Radzinski and J. B. Matson, J. Polym. Sci., Part A: Polym. Chem., 2017, 55, 2865.

10 A. Kerr, M. Hartlieb, J. Sanchis, T. Smith and S. Perrier, Chem. Commun., 2017, 53, 11901.

11 S. Shanmugam, J. Cuthbert, T. Kowalewski, C. Boyer and K. Matyjaszewski, Macromolecules, 2018, 51, 7776.

12 J. Tanaka, S. Häkkinen, P. T. Boeck, Y. Cong, S. Perrier, S. S. Sheiko and W. You, Angew. Chem., 2020, 59, 7203.

13 B. S. Sumerlin, ACS Macro Lett., 2012, 1, 141.

14 T. G. Floyd, S. Häkkinen, S. C. L. Hall, R. M. Dalgliesh, A.-C. Lehnen, M. Hartlieb and S. Perrier, Macromolecules, 2021, 54, 9461. 
15 M. Chen, M. Zhong and J. A. Johnson, Chem. Rev., 2016, 116, 10167.

16 S. Perrier, Macromolecules, 2017, 50, 7433.

17 G. Wang and J. Huang, Polym. Chem., 2014, 5, 277.

18 K. Skrabania, A. Miasnikova, A. M. Bivigou-Koumba D. Zehm and A. Laschewsky, Polym. Chem., 2011, 2, 2074.
19 R. N. Carmean, C. A. Figg, G. M. Scheutz, T. Kubo and B. S. Sumerlin, ACS Macro Lett., 2017, 6, 185.

20 M. G. Fröhlich, P. Vana and G. Zifferer, Macromol. Theory Simul., 2007, 16, 610.

21 M. M. Nardai and G. Zifferer, Polymer, 2013, 54, 4183.

22 M. G. Fröhlich, M. M. Nardai, N. Förster, P. Vana and G. Zifferer, Polymer, 2010, 51, 5122. 